Check for updates

Cite this: RSC Adv., 2018, 8, 37092

15th October 2018

Accepted 30th October 2018

DOI: $10.1039 / \mathrm{c} 8 \mathrm{ra0} 8536 \mathrm{f}$

rsc.li/rsc-advances

\section{A novel switchable-hydrophilicity, natural deep eutectic solvent (NaDES)-based system for bio-safe biorefinery}

\author{
Giorgia Sed, (D) ${ }^{a}$ Agnese Cicci, (D) ${ }^{b}$ Philip G. Jessop (iD) ${ }^{c}$ and Marco Bravi (iD *a
}

A switchable-hydrophilicity solvent system, consisting of a fatty acid-based natural deep eutectic solvent (NaDES), complemented by a bio-friendly dilute amine solution, has been introduced. The potential of the most benign switchable solvent system has been characterised in microalgae biorefining according to the recently proposed 'Circular Extraction' scheme.
"Biorefining", "market value" and "destination-neutrality" are almost inseparable concepts. "Biorefining", the operation consisting of separating the individual compounds of biomass, such as microalgae, aims at existing or new market segments, thus implying the concept of "market value", the amount of money that can be obtained from a given assembly of compounds. However, certain compounds, or assemblies thereof, may have multiple markets (e.g., biomaterials, detergents, food, feed, pharma, biofuel, and fertilisers, to just name a few key ones), where similar assemblies are subjected to different regulations and may be accordingly, differently valued. The term "destination-neutrality" denotes the possibility that a compound or an assembly may meet the restrictions entailed by the regulations of different markets; this is a key concept for creating maximum value for microalgal-derived products.

Switchable hydrophilicity solvents (SHS) are a new class of solvents that are able to change their nature from hydrophobic to hydrophilic and vice versa. ${ }^{1}$ So far, SHS systems have been created by biphasic systems composed of a hydrophobic liquid organic base and an aqueous layer. Upon addition of $\mathrm{CO}_{2}$, the liquid base becomes protonated and the resulting bicarbonate salt is fully miscible with water, converting the entire mixture into a single phase. Common SHS functional groups include alkylated amidines or secondary and tertiary amines that act as liquid bases to deprotonate carbonic acid or hydrated $\mathrm{CO}_{2} \cdot{ }^{2}$ Recently, Chen et al. described a switchable hydrophilicity system where the solvent is a fatty acid and the hydrophilic phase to make it switch is based on a dilute aqueous solution of a water-soluble amine. ${ }^{3}$ The switching mechanism, in this case, is different from that described above, in that the amine is able to create a complex with the fatty acid, so that the oily phase is entirely dissolved into the watery phase and the whole system

${ }^{a}$ Dept. Chemical Engineering, Materials, Environment, University "La Sapienza”, Via Eudossiana 18,00184, Rome, Italy.E-mail: marco.bravi@uniroma1.it

${ }^{b}$ BIO-P s.r.l., Via di Vannina 88, 00156, Rome, Italy

${ }^{c}$ Dept. Chemistry, Queen's University, Kingston, Ontario, K7L 3N6, Canada becomes hydrophilic. Addition of $\mathrm{CO}_{2}$ reprotonates the carboxylate anion, causing the hydrophobic carboxylic acid to phase separate from the aqueous phase. Switchable hydrophilicity solvents have been used in their hydrophobic form to extract hydrophobic solutes, such as oil from soybean flakes ${ }^{4}$ and microalgae, ${ }^{5}$ astaxanthin from microalgae, ${ }^{6}$ phenols from lignin-derived bio-oils ${ }^{7}$ and herbicides from water samples. ${ }^{8}$ In all of these studies the focus is limited to the extraction of only one compound or fraction from the biomass (or the liquid phase), the switching serving the purpose of separating that compound or fraction from the solvent. The SHS in the switched hydrophilic state is not used and must therefore be brought back to the initial hydrophobic condition prior to further use, so that the hydrophilic state of the SHS is necessary for the overall process but useless as far as extraction is concerned.

In our previous work we discussed the possibility of increasing the extraction of microalgal biomass components by exploiting both the native form of the SHS and the hydrophilic form obtained after the switching process, thereby increasing the overall utility of both the algae and the solvent. ${ }^{9}$ We also showed the power of this approach in contributing to the biomass fractionation into the main classes of biologicallyrelevant substances, and the entailed opportunity for optimising this fractionation by adopting the "forward-mode" (carrying out the extraction first by using the hydrophobic form of the SHS, and then the hydrophilic form) or the "backward-mode" (carrying out the extraction first by using the hydrophilic form of the SHS, and then switching back to the hydrophobic form for solvent recovery and then extraction of hydrophobic components) that can be adopted for the overall solid-liquid extraction unit operation (Fig. 1). It should be noted that Fig. 1 refers only to conceptual facts and does not care about their time sequence. Square and circle mean "hydrophilic step" and "hydrophobic step". Arrows tell the reader what goes in and out, without caring about time. The curved arrows going from the 


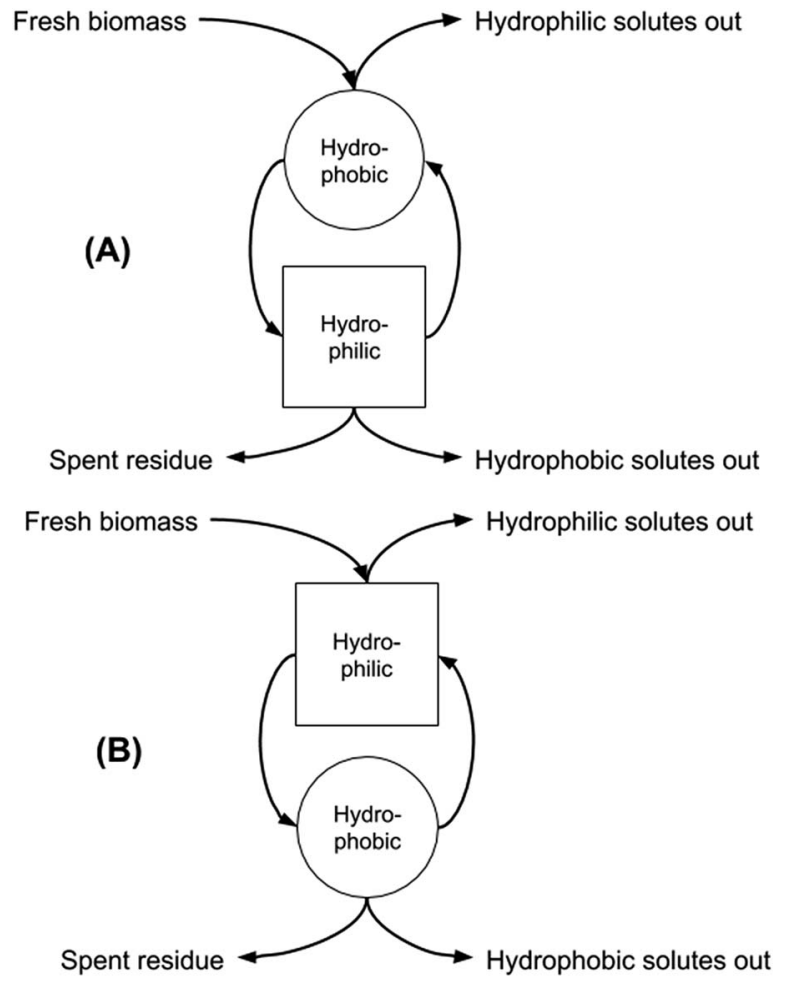

Fig. 1 Sequential two-stage extraction in forward (hydrophobic first, A) and backward (hydrophilic first, B) modes.

circle to the square and vice versa indicate that the hydrophobic phase becomes hydrophilic and vice versa.

In this work, a new SHS system with a substantial novelty is introduced in that it is based on a natural, deep eutectic solvent (NaDES) made of fatty acids and a weak amine water solution (Fig. 2). The NaDES which was adopted here is the mixture of octanoic acid and dodecanoic acid described by Florindo et al. and which the original inventors used only in its native state, as often done for NaDES systems. ${ }^{10}$ This NaDES is highly hydrophobic and exhibits a solidification temperature of $9{ }^{\circ} \mathrm{C}$ (compared to $16{ }^{\circ} \mathrm{C}$ and $43.8^{\circ} \mathrm{C}$ of the individual acids), so that it is normally in the liquid state at ambient temperature. This system was complemented by a dilute $(5 \%)$ aqueous solution of Jeffamine D-230, as described by Chen et al. ${ }^{3}$ It should be noted

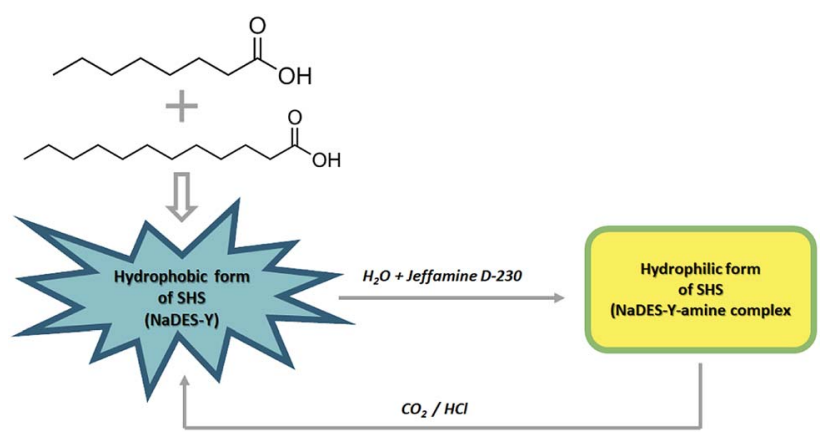

Fig. 2 NaDES-Y switching system, from hydrophobic form to hydrophilic and vice versa. that, while a single fatty acid solvent system suffers from high solidification temperature, the fatty acid-derived NaDES is more favourable in this respect. In the following, we will call the fatty acid deep eutectic solvent simply NaDES-Y, and the associated Jeffamine D-230 solution "amine solution", for brevity.

In the present work, we demonstrate that NaDES-Y: (1) can be made to switch, thus forming a hydrophilic phase; (2) can be made to switch back, with appropriate means, thus returning to the initial hydrophobic state; (3) can be used to solubilise triacylglycerides, and that these ones can be recovered upon switching the SHS to its hydrophilic form; (4) can be used to extract the biological fractions (proteins, carbohydrates and lipids) making up biomass. This latter part of the work was carried out according to the "Circular Extraction" paradigm" in two subsequent steps and in both the "forward-mode" and "backward-mode": (4.1) a synthetic matrix representing the microalgal biomass, made of selected proteins, lipids, carbohydrates, and water, was extracted by NaDES-Y and the extraction streams were characterised; (4.2) a sample of the adopted, wet microalgal biomass was extracted by NaDES-Y and the extraction streams were characterised.

In the first part of the experiment, $5 \mathrm{~mL}$ of NaDES-Y were mixed with the amine solution, in the volume ratio $1: 13$, and thoroughly mixed, thus obtaining a single, hydrophilic phase and demonstrating that the NaDES-Y can be switched in situ.

In the second part of the experiment, $\mathrm{CO}_{2}$ was bubbled into the mixture, thus acidifying it, reprotonating the carboxylate anions of the NaDES-Y and causing a phase splitting between the NaDES-Y and the amine solution. We have ascertained that the hydrophobic phase that separates from this phase splitting is still the original NaDES-Y by checking that its solidification temperature is unchanged, thus demonstrating that the NaDESY can also be switched-back in situ. It should be noted that the reversal of the system to the initial split-phase state can be obtained not only by injecting $\mathrm{CO}_{2}$ but also by acidification with strong acids, such as $\mathrm{HCl}$, although the use of the latter would likely increase the overall environmental impact of the method and would not be reversible, e.g. upon flushing with a gas stream such as air.

In order to test the suitability of this method for lipid solubilization and release, sunflower oil was used as a model system for triacylglycerols as in Jessop et al. ${ }^{11} 5 \mathrm{~mL}$ of NaDES-Y and $1 \mathrm{~mL}$ of sunflower oil were mixed. A single phase was obtained. Upon adding the aqueous amine solution and mixing, a phase split was produced between a hydrophilic phase consisting of the water and ammonium carboxylate salts of the hydrophilic NaDES-Y, and a hydrophobic phase made by sunflower oil.

Prior to the fourth part of the work, microalgal biomass of Scenedesmus dimorphus (UTEX 1237) was cultivated in our laboratory and then compositionally assessed. Carbohydrates and proteins were quantified colorimetrically with spectrophotometry; total carbohydrates were quantified by the Dubois assay $^{\mathbf{1 2}}$ and total proteins were quantified by the Lowry assay. ${ }^{\mathbf{1 3}}$

A model matrix was prepared by blending starch $(0.38 \mathrm{~g})$, glucose $(0.091 \mathrm{~g})$, casein $(0.177 \mathrm{~g}$, adopted as a widely available hydrophobic protein), albumin (0.158 g, adopted as a widely available hydrophilic protein), sunflower oil (0.09 $\mathrm{g}$ adopted as 
a widely available triacylglycerides pool), soy lecithin $(0.04 \mathrm{~g}$ adopted as a widely available phospholipids pool to represent cellular membranes) and water $(0.75 \mathrm{~g}$ adopted to represent intrinsic and extrinsic water). The composition of this matrix was chosen to simulate the measured composition of $S$. dimorphus and the water content of "wet" microalgal biomass separated by centrifugation of the cultured suspension (20 minutes at $2600 \mathrm{~g}$ ). Subsequently, the extraction of the model matrix was carried out with NaDES-Y, in its native hydrophobic state. The model matrix (1.58 g) was thoroughly mixed with $30 \mathrm{~mL}$ of NaDES-Y and agitated for $24 \mathrm{~h}$ in the presence of glass beads. Then agitation was interrupted and any insoluble material was separated and stored for the second-stage extraction. The aqueous amine solution was added to the homogeneous NaDES-Y thus causing the phase split that expels oily fractions. The now-hydrophilic solvent was then collected and used to further treat the insoluble material that had been stored at the end of the first extraction stage, again by prolonged thorough mixing. Finally, any still insoluble material was removed and the liquid was switched back to its initial state. By following the two extraction stages described here, the complete "forward-mode", dual-stage circular extraction was complete, and the extraction capability of the SHS was assessed by characterising both the supernatant streams and the solid residuals.

In a separate experiment, the extraction order was reversed, thus following what was named the "backward-mode" circular extraction in our previous work. ${ }^{9}$ In this case the model matrix was first treated with NaDES-Y that had been already mixed with the amine solution, thus becoming hydrophilic, producing an aqueous liquid phase and an intermediate solid residue that was stored. After addition of $\mathrm{CO}_{2}$ to the liquid phase, triggering a phase split, the supernatant (the hydrophobic NaDES-Y), was decanted from the aqueous phase that contained the extracted hydrophilic components from the model biomass mixture. The recovered NaDES-Y was then used to extract hydrophobic components from the intermediate solid residue, thus obtaining a second extract and a final insoluble residue. All streams were compositionally assessed as before, thus completely characterising the backward-mode dual-stage extraction in the synthetic matrix.

All of the above extraction procedures, both in forward mode and in backwards mode, were also performed on the microalgal biomass rather than the synthetic matrix. First, however, we investigated the capability of the solvent to break the cell wall by agitating a microalgal cells-in-solvent suspension for $24 \mathrm{~h}$. The very poor yield in a subsequent forward-mode extraction indicated that the mildly acidic $\mathrm{pH}(\sim 2)$ of the solvent was unable to cause cell rupture by itself, and that an additional cell disruption method would be necessary. Further extractions were performed in the presence of glass beads, which greatly improved the extraction of lipids, and moderately improved the extraction of proteins and carbohydrates. A further provision to boost the extraction of the lagging fractions was the application of microwaves ( $90 \mathrm{~s}$ at $300 \mathrm{~W}$ in a household oven). However, when carrying out the "forward-mode" extraction, we realised that the combined effect of microwaving (which implies heating dipoles such as water) in the presence of an acidic $\mathrm{pH}$ (imparted by the fatty acids) caused an extensive degradation of chlorophyll (to pheophytin), a well-known undesired outcome of misperformed sterilisation processes of vegetables in the food industry. The solution, here, came from the very solution adopted in vegetables sterilisation, that is inducing a mild alkalinity in the food mass that must be sterilised. However, in circular extraction, it is not necessary to actually perform any alkalinisation, because it is sufficient to adopt the "backward-mode" instead of the forward mode extraction. Indeed, achieved the desired outcome of higher yields without extensive degradation of chlorophyll.

The quantitative results of parts 4.1 and 4.2 are reported in Fig. 3 and 4 (model system, forward- and backward-mode), 5, 6 and 7 (microalgal biomass, various extraction implementations in forward- and backward-mode).

The reported results should be interpreted as follows: during the forward-mode extraction (Fig. 2), 41\% of the carbohydrates contained by the model matrix were dissolved by NaDES-Y, which has a hydrophobic character. Switched NaDES-Y, which has a hydrophilic character, managed to extract a further $26 \%$ of the initial carbohydrates that had remained in the matrix residue after the first stage of the forward-mode extraction. The total carbohydrates extraction reached therefore $67 \%$. It should be noted that the total extraction is split between two streams; therefore, their recovery should be performed through both the hydrophobic-to-hydrophilic switching and the hydrophilic-tohydrophobic switching. The backward-mode extraction (Fig. 2) has different extraction yields, but the same concept holds.

Synthetic matrix extractions results (Fig. 3 and 4) show that, as far as total extraction is concerned, separate consideration may be made for proteins, carbohydrates and neutral lipids, and their distribution in the two subsequent extract streams obtained. Neutral lipid extraction is almost quantitative whatever orientation (forward or backward) of the operation is adopted and protein extraction is nearly quantitative, with a slight preference for backward rather than forward orientation (99\% vs. 93). In the case of carbohydrates, a more marked difference between forward and backward orientation is observed (66\% vs. $86 \%$ ), again with an advantage of the backward mode over the forward mode. From the point of view of fraction distribution between extracts, neutral lipids are exclusively obtained from the hydrophobic extract and are absent in

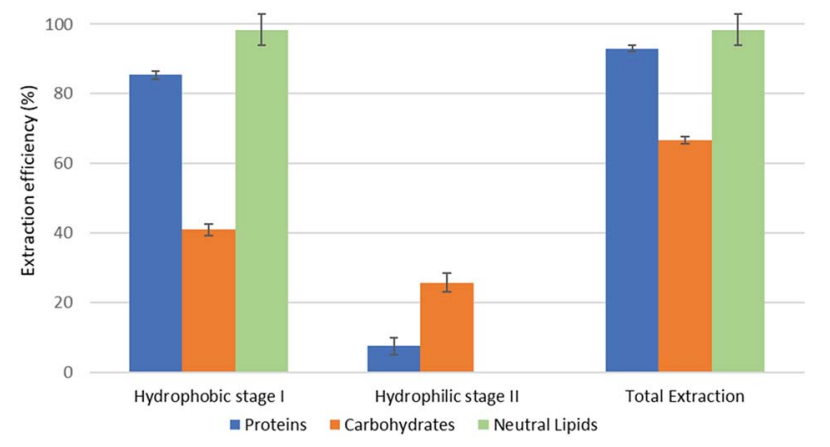

Fig. 3 Fractional extraction efficiencies on model system extracted in forward-mode. 


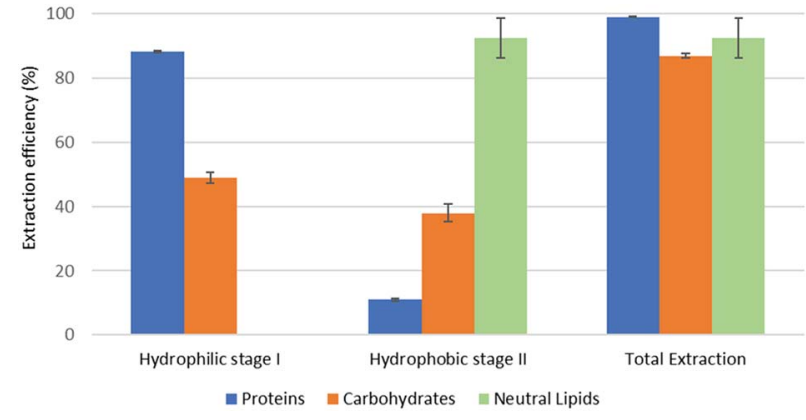

Fig. 4 Fractional extraction efficiencies on model system extracted in backward-mode.

the hydrophilic extract, whatever the orientation of the operation.

Protein extraction, on the other hand, appears to be split between the two subsequently staged extractions in the opposite way. The extraction occurring in the first place picks up a large fraction of the proteins ( $85 \%$ if it is hydrophobic, $88 \%$ if it is hydrophilic), leaving a much smaller fraction to be accomplished by the extraction performed subsequently $(8 \%$ if it is the hydrophilic follow-up of a forward-mode extraction, $11 \%$ if it is the hydrophobic follow-up of a backward-mode extraction). Carbohydrates extraction shows a behaviour that is similar, albeit less marked, to that of proteins. The extraction occurring first picks up a comparatively larger fraction of the proteins originally contained in the matrix ( $41 \%$ if it is hydrophobic, $49 \%$ if it is hydrophilic), leaving a somewhat smaller fraction for the extraction coming later $(26 \%$ if it is the hydrophilic followup of a forward-mode extraction, $38 \%$ if it is the hydrophobic follow-up of a backward-mode extraction).

While the result observed for neutral lipids was expected, explanation for the behaviour of the proteins is less intrinsically clear. Proteins are equally distributed between hydrophilic- and hydrophobic-character in the synthetic matrix. However, well above the available amount of protein matching the type of solvent (hydrophilic vs. hydrophobic) is extracted in the extraction stage coming first. This might be due to the formation of micellar systems created by the phospholipids that were added to represent cell membranes and eased the extraction, pretty much as it occurs in micelle-assisted protein recovery techniques; ${ }^{14}$ indeed, the rough solubility of casein (representing hydrophobic proteins) in native (hydrophobic) NaDES-Y is $0.24 \mathrm{~g} \mathrm{~L} \mathrm{~L}^{-1}$, while the calculated casein concentration in our hydrophobic extract (first stage of the forward-mode extraction of the synthetic matrix) is $4.60 \mathrm{~g} \mathrm{~L}^{-1}$; the rough solubility of albumin (representing hydrophilic proteins) in native NaDES-Y is $0.22 \mathrm{~g} \mathrm{~L}^{-1}$, while the calculated albumin concentration in our hydrophobic extract is $4.00 \mathrm{~g} \mathrm{~L}^{-1}$. Analogously, the rough solubility of casein in the hydrophilic form of NaDES-Y is $0.06 \mathrm{~g}$ $\mathrm{L}^{-1}$, while the calculated casein concentration in our hydrophilic extract (from the first stage of the backward-mode extraction of the synthetic matrix) is $4.35 \mathrm{~g} \mathrm{~L}^{-1}$; the rough solubility of albumin in hydrophobic-to-hydrophilic-switched NaDES-Y is $0.52 \mathrm{~g} \mathrm{~L} \mathrm{~L}^{-1}$, while the calculated albumin concentration in our hydrophilic extract is $4.45 \mathrm{~g} \mathrm{~L}^{-1}$. Carbohydrates are not only more extensively extracted in backwardmode in the term of the total amount, but they are also dissolved more in each partial step of it (both the first and the second step show a $+10 \%$ increase).

The results of part 4.2 of the work show that the method of cell disruption has a strong effect on the yield of extract. As mentioned above, barely agitating a microalgal suspension in either hydrophobic or pre-switched NADES-Y does not succeed in ensuring a significant extraction yield most likely due to the unbroken cell wall hindrance and was not investigated further (the extraction efficiency resulted to be $2.0 \% \pm 2.6 \%$ for proteins, $5.1 \% \pm 4.4 \%$ for carbohydrates, with only traces of neutral lipids). When bead beating was added, a significant improvement to lipid yield was recorded during the hydrophobic step of the forward-mode extraction (88\%) (Fig. 5), while protein and carbohydrate extraction was essentially unchanged. However, during the subsequent hydrophilic stage of the forward-mode extraction, a further $32 \%$ and $25 \%$ of the microalgal proteins and carbohydrates, respectively, were picked up from the microalgal matrix, thus leading to an overall extraction of $36 \%$ of the original proteins and $33 \%$ of the original carbohydrates. When reversing the extraction (backward-mode) (Fig. 6), 17\% and 8\% of the original proteins and carbohydrates, respectively, were dissolved during the hydrophilic stage; after hydrophilic-to-hydrophobic switching the solvent, a further 28\% (proteins) and 17\% (carbohydrates) extraction was possible, thus attaining an overall extraction ratio of $45 \%$ and $25 \%$, respectively, of the original proteins and carbohydrates of the microalgal matrix. Lipids were extracted exclusively in the hydrophobic stage, and total extraction did not vary appreciably between the forward-mode and the backward-mode conduite of the dual-stage extraction. It is therefore apparent that beads beating ensures a fair yield in lipids, while proteins and carbohydrate extraction is promoted to a lesser degree. The second cell disruption technique tested, microwave-assisted extraction, could only be applied to the hydrophilic phase and, although it could also have been limited to the hydrophilic stage of the forward-mode extraction, in this study it was limited to the (hydrophilic) first stage of the

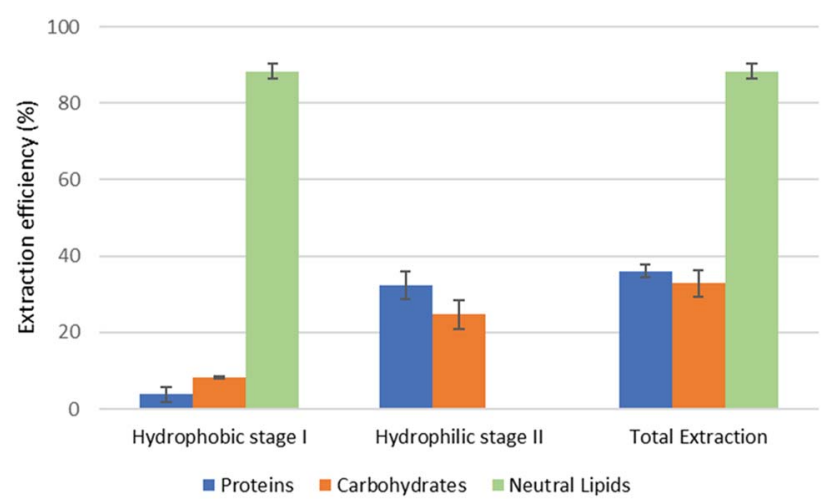

Fig. 5 Fractional extraction efficiencies on bead beating-assisted microalgal suspension extraction in forward-mode. 


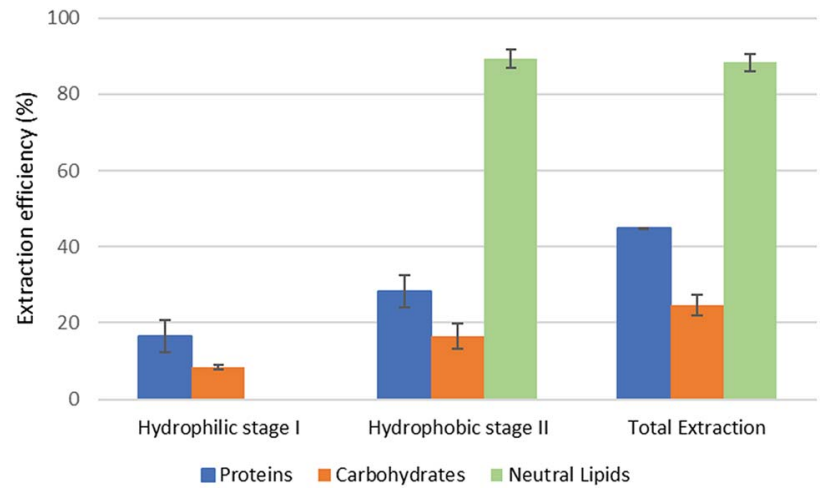

Fig. 6 Fractional extraction efficiencies on bead beating-assisted microalgal suspension extraction in backward-mode.

backward-mode extraction. Results (Fig. 7, "microwave-assisted microalgal suspension extraction") show a clear improvement in extractability of proteins during the first (hydrophilic) stage of the extraction $(54 \%$ vs. $17 \%$ in the first stage of the beadbeaten extraction), while protein extraction in the second stage (hydrophobic, un-microwaved) became less efficient $(12 \%$ vs. $28 \%$ in the first stage of the bead-beaten extraction). Overall protein extraction ratio, however, jumped from $45 \%$ to $72 \%$. Carbohydrates resembled the proteins behaviour in a mirrored way: their extractability during the first (hydrophilic) stage of the extraction was only slightly promoted by the microwave treatment $(18 \%$ vs. $8 \%$ in the first stage of the bead-beaten extraction), while in the second stage (hydrophobic, unmicrowaved) their extraction was boosted $(41 \%$ vs. $17 \%$ in the second stage of the bead-beaten extraction). Overall carbohydrate extraction, therefore, was significantly promoted, from $25 \%$ to $59 \%$.

In combination with a cell wall rupturing technique, therefore, the anticipated extraction potential toward biologic fractions recorded during synthetic matrix extraction experiments was well confirmed on microalgal matrix for lipids, while a $20-$ $60 \%$ lower extraction was recorded for proteins and carbohydrates, most likely due to residual diffusional hindrances and inter-fraction cross-link effects which are the fundamental (non

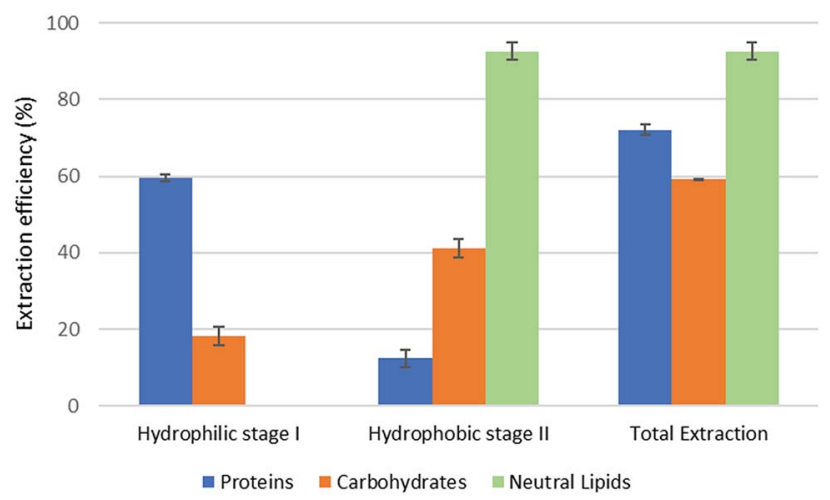

Fig. 7 Fractional extraction efficiencies on microwave-assisted microalgal suspension extraction in backward-mode. compositional) difference between the synthetic and the natural matrix.

Compared to the case study reported in the original 'Circular Extraction' article, in the present work extraction ratios are broadly comparable in the cases where NaDES-Y extraction is assisted by some means which is capable of breaking cell walls. However, depending on the type of cell disruption technique deployed, the results may be inferior, comparable, or superior to those obtained with DMCHA. Thus, bead beating-assisted extraction comes close to DMCHA extraction for proteins ( $36 \%$ vs. $41 \%$ ) and lipids ( $88 \%$ vs. $96 \%$ ) but is less efficient with carbohydrates (33\% vs. $51 \%)$ in forward-mode extraction. In backward-mode extraction NaDES-Y, again, comes close to DMCHA efficiency for proteins ( $45 \%$ vs. $52 \%)$ and lipids (89\% vs. $93 \%$ ), while carbohydrate extracting power is half that of DMCHA (25\% vs. $50 \%)$. If microwaved extraction is used the extraction with NaDES-Y improves significantly, as noted before, and thus NaDES-Y ranks as the best extracting medium for proteins ( $72 \%$ vs. $52 \%)$ and carbohydrates (59.3\% vs. 51.4\%) and comes very close to DMCHA efficiency for lipids ( $93 \% v s$. $96 \%)$.

From a biorefinery application perspective, adopting the bead beaten-assisted forward-mode extraction may warrant extracting lipids $(88 \%)$ during the hydrophobic stage while proteins and carbohydrates extraction task is covered by the hydrophilic stage. On the other hand, by adopting the microwave-assisted extraction backward-mode extraction, during the hydrophilic stage NaDES-Y is able to extract more proteins than DMCHA ( $54 \%$ vs. $50 \%$ ), with less contamination by co-extracted carbohydrates ( $18 \%$ instead of $47 \%$ ), while the subsequent hydrophobic stage can extract more carbohydrates than DMCHA-based would ( $41 \%$ vs. $4 \%$ ) in the same stage. While it may be observed that lipids would be co-extracted, this would not actually cause any recovery problem, given that the subsequent hydrophobic-to-hydrophilic form switch bring their separation about as shown in part 3 of the present results.

\section{Conclusions}

NaDES systems are often touted as 'designer solvents' because they offer the possibility of regulating their hydrophilicity by adding water. However, the range of hydrophilicity variation is modest and can be used, at most, to optimise the extraction of certain solutes. Hydrophilicity cannot be reversed the way SHS allow. In this work, we have devised a way to go back and forth from the hydrophobic to the hydrophilic behaviour and vice versa by reversibly modifying a hydrophobic NaDES with a lean amine in water solution, so that its hydrophobic components are complexed and the resulting solution is hydrophilic. This finding describes therefore the first switchable NaDES-based solvent described in the open literature, although the exact nature of the hydrophilic phase (and, in particular, whether the supramolecular structure is retained, or is destroyed and then reconstituted upon hydrophilic-to-hydrophobic switch) remains to be clarified. The modest amine content of the water solution and the very low toxicity of the adopted amine also duplicate this as a practically bio-safe SHS (at the working 
concentration of Jeffamine in the hydrophilic phase, the estimated lethal dose is $>50 \mathrm{~g} \mathrm{~kg}^{-1}$ ). Together, the two findings mark a significant step toward the identification of a destination-neutral biorefining tool for the obtainment of biologic fractions from biomasses with a wide range of potential market applications. From an application point of view, this solvent is not able to fracture cell walls, and thus requires a complementary step to achieve this (such as bead beating or microwaving). However, NaDES-Y assisted with a complementary cell-breaking step features an extraction performance which is nearly equal to that of DMCHA for all the examined biological fractions (with beads beating) and may be even higher than that of DMCHA for proteins and carbohydrates (in microwave-assisted extractions). NaDES-Y, with the combined freedom of choice of forwardmode or backward-mode circular extraction and of the (required) cell wall fracturing provision, may supply a powerful tool in the hands of the biochemical process engineer. Anticipated future work includes drafting a roadmap toward biologically safe and process-wise feasible SHS circular extraction of microalgal biomass by presenting results obtained with a variety of different SHS in order to identify that which best combines efficacy in the two extractions with minimum environmental impact.

\section{Conflicts of interest}

The authors declare that there are no conflicts of interest.

\section{Acknowledgements}

The authors gratefully acknowledge the collaboration of Martina Buldo, for her assistance in the experiments during her graduation thesis work. The authors owe a special mention also to Christopher Paiewonsky, for his contribution to our former efforts in NaDES-based SHS development.

\section{Notes and references}

1 P. G. Jessop, L. Kozycz, Z. Ghoshouni Rahami, D. Schoenmakers, A. R. Boyd, D. Wechsler and A. M. Holland, Green Chem., 2011, 13, 619-623.

2 J. R. Vanderveen, J. Durelle and P. J. Jessop, Green Chem., 2014, 16, 1187-1197.

3 Q. Chen, L. Wang, G. Ren, Q. Liu, Z. Xu and D. Sun, J. Colloid Interface Sci., 2017, 504, 645-651.

4 P. G. Jessop, L. Carrier, A. Robinson, C. J. Dürr and J. R. Hariani, Green Chem., 2010, 12(5), 809-814.

5 C. Samori, D. L. Barreiro, R. Vet, L. Pezzolesi, D. W. F. Brilman, P. Galletti and E. Tagliavini, Green Chem., 2013, 2, 353-356.

6 W. C. Huang, H. Liu, W. Sun, C. Xue and X. Mao, ACS Sustainable Chem. Eng., 2018, 6(2), 1560-1563.

7 D. Fu, S. Farag, J. Chaouki and P. G. Jessop, Bioresour. Technol., 2014, 154, 101-108.

8 G. Lasarte-Aragonés, R. Lucena, S. Cárdenas and M. Valcárcel, J. Sep. Sci., 2015, 38(6), 990-995.

9 A. Cicci, G. Sed, P. G. Jessop and M. Bravi, Green Chem., 2018, 20, 3908-3911.

10 C. Florindo, L. Romero, I. Rintoul, L. C. Branco and I. M. Marrucho, ACS Sustainable Chem. Eng., 2018, 6(3), 3888-3895.

11 P. G. Jessop, S. M. Mercer and D. J. Heldebrant, Energy Environ. Sci., 2012, 5(6), 7240-7253.

12 M. Dubois, K. A. Gilles, K. A. Hamilton, P. T. Rebers and F. Smith, Anal. Chem., 1956, 28(3), 350-356.

13 O. H. Lowry, N. J. Rosebrough, A. Lewis Farr and R. J. Randall, J. Biol. Chem., 1951, 193(1), 265-275.

14 Y. Liu, X. Dong and Y. Sun, Chin. J. Chem. Eng., 2008, 16(6), 949-955. 